\title{
Venetoclax approved to treat CLL patients regardless of genotype
}

T The approval of Bcl-2 inhibitor venetoclax was expanded by the US Food and Drug Administration in June 2018 to include the treatment of patients with chronic lymphocytic leukemia (CLL) or small lymphocytic leukemia (SLL), regardless of their genotype, who have received at least 1 prior therapy. ${ }^{1}$ It was previously approved in 2016 for the treatment of patients who had a chromosome $17 p$ deletion, which leads to loss of the tumor-suppressor gene TP53.

Approval was based on the positive results of the phase 3, randomized, multicenter, open-label MURANO trial in which 389 patients were randomized 1:1 to receive a combination of venetoclax and the CD20targeting monoclonal antibody rituximab (venetoclaxrituximab) or bendamustine in combination with rituximab (bendamustine-rituximab).

Eligible patients were 18 years of age or older, had been diagnosed with relapsed/refractory CLL that required treatment, had received 1-3 prior therapies (including at least 1 chemotherapy regimen), had an Eastern Cooperative Oncology Group performance status of 0 or 1 (on a 5 -point scale, with 5 indicating the greatest level of disability), and had adequate bone marrow, renal, and hepatic function.

Patients who had received prior bendamustine treatment were eligible for the trial provided they had experienced a duration of response of 24 months or longer. However, patients with transformed CLL, central nervous system involvement, prior treatment with allogeneic or autologous stem cell transplant, major organ dysfunction, other active malignancy, or who were pregnant or breastfeeding, were excluded from the study.

Patients in the venetoclax arm received a 5-week rampup schedule, followed by a dose of $400 \mathrm{mg}$ once daily for 24 months. Rituximab treatment started at the end of the venetoclax ramp-up period and was administered at a dose of $375 \mathrm{mg} / \mathrm{m}^{2}$ intravenously on cycle 1 day 1 and $500 \mathrm{mg} / \mathrm{m}^{2}$ on day 1 of cycles 2-6. In the control arm, patients received 6 cycles with the same rituximab dosing and schedule as the study group and bendamustine at a dose of $70 \mathrm{mg} / \mathrm{m}^{2}$ on days 1 and 2 of each 28 -day cycle.

The primary endpoint was progression-free survival (PFS), as assessed by an independent review committee over a median follow-up of 23 months. Median PFS

\section{What's new, what's important}

The significant improvement in PFS demonstrated in the MURANO trial in patients with CLL or SLL who were treated with the venetoclax-rituximab combination is highly encouraging for clinicians and patients alike. The findings were the basis for the approval of venetoclax for patients with previously treated relapsed/refractory CLL, regardless of genotype, in which patients were randomized to receive either the venetoclax-rituximab or bendamustine-rituximab combinations.

Patients in the venetoclax arm received a 5-week ramp-up schedule, then $400 \mathrm{mg}$ daily for 24 months. Rituximab was initiated at the end of the venetoclax ramp-up $(375 \mathrm{mg} / \mathrm{m} 2 \mathrm{IV}$ on day 1 of cycle 1 , and $500 \mathrm{mg} / \mathrm{m} 2$ on day 1 of cycles $2-6$ ). Controls received bendamustine at $70 \mathrm{mg} / \mathrm{m} 2$ on days 1 and 2 of each 28-day cycle, and 6 cycles with the same rituximab dosing and schedule as the study patients.

The primary endpoint was PFS assessed over a median follow-up of 23 months. Median PFS was significantly improved in the venetoclax arm (not yet reached vs 18.1 months in the bendamustine arm) and ORR and EFS were also better in the study group compared with controls (ORR: $92 \%$ and $72 \%$, respectively; 2-year EFS: $84.9 \%$ and $34.8 \%$ ). A trend toward improved 24 -month OS rate $(91.9 \%$ vs $86.6 \%)$ did not achieve statistical significance, nor did median OS.

The most common AEs with venetoclax were neutropenia, diarrhea, upper-respiratory tract infection, fatigue, cough, and nausea (grade $3 / 4$ neutropenia: $64 \%$ of patients; serious $A E s$ : $46 \%$ ). Serious infections occurred in $21 \%$ of patients; there were 10 treatment-related deaths in the venetoclax arm, and 11 in the bendamustine arm.

Warnings and precautions relate to the risk of tumor lysis syndrome, which is higher in patients with higher tumor burden or reduced renal function, or who receive strong or moderate CYP3A inhibitors or P-gp inhibitors during ramp-up. Preventive strategies would include hydration and antihyperuricemics, monitoring of blood chemistry and timely management of abnormalities, or dose interruption or adjustment as needed. Other warnings relate to neutropenia, immunization, and embryofetal toxicity.

- Jame Abraham, MD, FACP (abrahaj5@ccf.org)

Report prepared by Jane de Lartigue, PhD. JCSO 2018;16(5):e185-e187. C2018 Frontline Medical Communications. doi: https://doi. org/10.12788/jcso.0422 


\section{Mechanism of action: venetoclax}

\section{Blocking the cancer hall- mark of avoiding apop-}

tosis. Venetoclax is a smallmolecule inhibitor of the $\mathrm{Bcl}-2$ protein, the eponymous member of a family of proteins that play a central role in apoptosis, a form of cell death that clears unwanted or damaged cells and maintains tissue homeostasis. There are 2 major pathways involved in regulating apoptosis - the intrinsic and extrinsic pathways, which are triggered by signals that come from inside and outside the cell, respectively.

The Bcl-2 family of proteins predominantly regulate the intrinsic pathway and contain both anti-apoptotic and proapoptotic members that interact with one another to finely tune the signals that govern cell fate. In healthy cells, pro-apoptotic $\mathrm{Bcl}-2$ family proteins are bound by anti-apoptotic members ( $\mathrm{Bcl}-2$ among them), which helps to keep their activity in check.

In response to stimuli, such as DNA damage or defects in mitosis, some of the pro-apoptotic proteins (known as BH3-only proteins) are activated and bind to the anti-apoptotic proteins. This relieves their suppression on other pro-apoptotic proteins (known as effector proteins), particularly BAK and BAX. These proteins are then free to partner up and form small complexes that insert themselves into the mitochondrial membrane, creat- ing holes through which cytochrome $\mathrm{c}$ is able to escape.

In the cytoplasm, cytochrome $c$ drives the formation of the apoptosome, a protein complex that activates a family of protease enzymes - the caspases, which are key effectors of apoptosis, breaking down intracellular proteins.

The ability to evade apoptosis and thus continue to proliferate unchecked is a hallmark of cancer cells and, as a central regulator of apoptosis, $\mathrm{Bcl}-2$ is overexpressed in several different malignancies, including CLL and SLL. Thus, it represents a key therapeutic target. was significantly improved in the venetoclax arm (not yet reached versus 18.1 months in the bendamustine arm [HR, $0.19 ; P<.001])$. In addition, objective response rate (ORR) and event-free survival (EFS) also favored the venetoclax arm; ORR was $92 \%$ compared with $72 \%$, respectively, and 2 -year EFS was $84.9 \%$ compared with $34.8 \%$. There was also a trend toward improved 24-month overall survival (OS) rate (91.9\% vs 86.6\%), however this did not achieve statistical significance, nor did median OS.

The most common adverse events (AEs) in patients treated with venetoclax were neutropenia, diarrhea, upperrespiratory tract infection, fatigue, cough, and nausea. Grade 3/4 neutropenia occurred in $64 \%$ of patients, and serious AEs in $46 \%$ of patients. Serious infections occurred in $21 \%$ of patients, most commonly pneumonia. Ten deaths in the venetoclax arm were attributed to treatment, compared with 11 deaths in the bendamustine arm. ${ }^{2}$

The prescribing information details warnings and precautions relating to the risk of tumor lysis syndrome, which is increased in patients with higher tumor burden, reduced renal function, or in receipt of strong or moderate CYP3A inhibitors or P-gp inhibitors during the ramp-up stage. Patients should receive appropriate preventive strategies, including hydration and antihyperuricemics, blood chemistry should be monitored and abnormalities managed promptly, and dosing should be interrupted or adjusted as necessary.

Other warnings relate to neutropenia (complete blood counts should be monitored throughout treatment and 
venetoclax treatment interrupted or dose reduced for severe neutropenia, alongside possible use of supportive measures), immunization (live vaccines should not be administered before or during treatment or after treatment until B-cell recovery, and patients should be

\section{References}

1. US Food and Drug Administration website. FDA approves venetoclax for CLL or SLL, with or without 17p deletion, after one prior therapy. https://www.fda.gov/drugs/informationondrugs/approveddrugs/ucm610308.htm. Last updated June 8, 2018. Accessed July 29, 2018

2. Seymour JF, Kipps TJ, Eichhorst B, et al. Venetoclax-rituximab in advised of the potentially reduced efficacy of vaccines), and embryofetal toxicity (patients should be advised of the risks and the need for effective contraception during and after treatment). Venetoclax is marketed as Venclexta by Genentech. ${ }^{3}$

relapsed or refractory chronic lymphocytic leukemia. N Engl J Med. 2018;378:1107-1120.

3. Venclexta (venetoclax tablets) for oral use. Prescribing information. Genentech USA, Inc. https://www.accessdata.fda.gov/drugsatfda_docs/label/2016/208573s000lbl.pdf. Last updated June 2018. Accessed July 29, 2018. 